\title{
Intelligent Ray-Tracing: an efficient indoor ray propagation model
}

\author{
Ahmed Wasif Reza ${ }^{1 a}$, Kaharudin Dimyati ${ }^{2}$, \\ Kamarul Ariffin Noordin ${ }^{1}$, and Md. Sumon Sarker ${ }^{1}$ \\ ${ }^{1}$ Faculty of Engineering, Department of Electrical Engineering \\ University of Malaya, 50603 Kuala Lumpur, Malaysia \\ ${ }^{2}$ Faculty of Engineering, National Defence University of Malaysia \\ Sg. Besi Camp, 57000 Kuala Lumpur, Malaysia \\ a)awreza98@yahoo.com
}

\begin{abstract}
This study proposes an efficient and accelerated Intelligent Ray-Tracing (IRT) algorithm based on Binary Angle Division (BAD) technique for radio signal prediction in indoor area. The intelligent features of the proposed IRT can skip the processing of the unnecessary signals based on the invalid region and reduce the number of candidate objects (obstacles) as well as their edges while performing ray-object intersection tests, which can make the algorithm faster as well as more accurate. The obtained results are compared with the existing indoor ray propagation methods to prove the superiority of the proposed IRT technique in terms of both computational efficiency and accuracy of signal prediction.
\end{abstract}

Keywords: Ray-tracing, radio signal prediction, indoor propagation Classification: Electromagnetic theory

\section{References}

[1] M. S. Sarker, A. W. Reza, and K. Dimyati, "A novel ray-tracing technique for indoor radio signal prediction," J. Electromagnetic Waves and Applications, vol. 25, pp. 1179-1190, 2011.

[2] M. F. Iskander and Z. Yun, "Propagation Prediction Models for Wireless Communication Systems," IEEE Trans. Microw. Theory Techn., vol. 50, no. 3, pp. 662-673, March 2002.

[3] R. Hoppe, G. Wolfle, and F. M. Landstorfer, "Measurement of building penetration loss and propagation models for radio transmission into buildings," IEEE Vehicular Technology Conference, vol. 4, pp. 2298-2302, 1999.

[4] Z. Raida, Z. Lukes, and J. Lacik, "On Using Ray-Launching Method for Modeling Rotational Spectrometer," Journal: Radioengineering, vol. 17, no. 2, pp. 98-107, 2008. 


\section{Introduction}

The use of wireless communication in indoor area associated with Personal Communication Systems (PCS) is exploding rapidly. Since practical measurements are interpreted as costly service for the consumer, there is an increasing demand for an efficient and accurate simulation-based tool that will facilitate the propagation prediction of radio signal in indoor environment [1]. Therefore, the purpose of this study is to introduce a highly accurate and very fast propagation prediction model, such as Intelligent Ray-Tracing (IRT) algorithm based on Binary Angle Division (BAD). The Ray-Tracing (RT) is used to predict the trajectory of the signal while triangular geometry is used to define the propagation path of the radio signal emanated from the transmitter. The BAD [1] equally divides the angular distance between two consecutive signals emanated from the transmitter to reduce the computation time. In contrast with [1], the smart features of the proposed IRT involve skipping the processing of the unnecessary signals based on the invalid region and reducing the number of objects (obstacles) of the intersection test to make the algorithm faster and accurate in terms of signal prediction. The superiority of the proposed IRT is proved by comparing with other ray propagation models, such as RT [1,2] and ray-launching [3, 4]. The design details including the results and analysis of the proposed IRT will be discussed in Sections 2 and 3, respectively. Finally, a conclusion will be presented in Section 4 .

\section{Procedure}

In this study, the proposed IRT technique has been developed using C\# 2008 under Microsoft Dot Net Framework 3.5. The C\# is an object oriented programming language that can be easily used to perform an intersection test for a ray and an object.

For ease of understanding, this study considers two dimensional (2D) space for a typical indoor environment and presents a simple and accurate guideline for tracing the trajectory of the signal between the transmitter $(T x)$ and the receiver $(R x)$ as shown in Fig. 1 (a), where the co-ordinate system starts from the top left corner. For this case, a ray TxS1 launched at angle $\theta$ forms a triangle $\Delta T x A 1 S 1$ where $A 1 S 1=H$ is the normal. Based on the triangular geometry, the value of $H$ can be calculated by $H=\tan (\theta) *(X$ value of $T x)$. Thus, the end point of the ray $T x S 1$ is $S 1(X, Y)=S 1(0, Y$ value of $T x-H)$. Then, the nearest intersection point is $R 1$, where the ray hits an edge of the object is calculated. Moreover, while doing intersection test, the unnecessary test for signal-object interaction can be ignored as shown in Fig. 1 (b) to accelerate the overall execution time as follows:

(i) From the current transmission point of the ray, divide the space into four quadrants and find all objects that are in the same quadrant with the ray. The lines from the transmitter $(T x)$ refer to the rays. To check whether any object is in the first quadrant, make sure that only top right corner of 
(a)

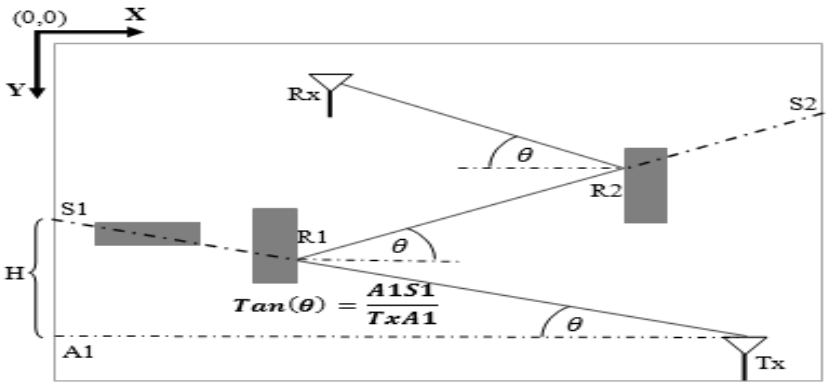

(b)

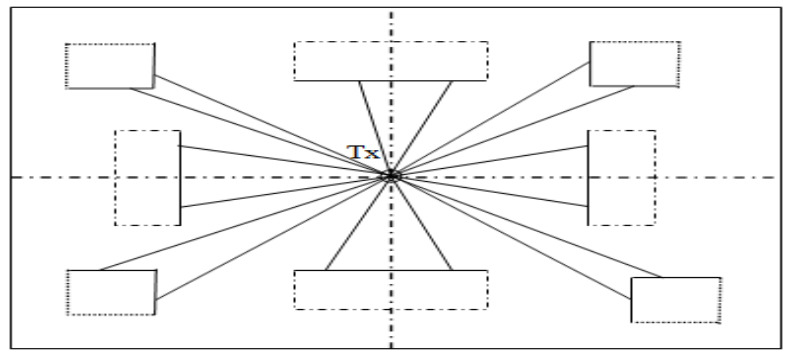

Fig. 1. (a) Illustration of determining the trajectory of the signal (b) Valid intersections between the rays and the edges of the objects.

the object is in the first quadrant. Again, to check whether any object is in the second quadrant, make sure that only top left corner of the object is in the second quadrant. Similarly, to check whether any object is in the third or fourth quadrant, bottom left corner or bottom right corner, respectively has to be in the corresponding quadrant. In this way, it is possible to reduce the number of objects while doing ray-object intersection tests.

(ii) An object can be considered as consisting of four edges including four corners. All edges of an object do not take part while doing ray-object intersection tests. For example, if an object is in the first quadrant with respect to the current component of the trajectory of the ray, then the possible edges for intersection test are the left and bottom edges. If top left corner of the object is in the first quadrant, then intersection between the left edge of the object and the ray is possible. Again, if the bottom right corner is in the first quadrant, then intersection between the bottom edge of the object and the ray is possible. Other edges (top and right) are ignored entirely. Based on this concept, all the valid intersections have been shown in Fig. 1 (b). Here, vertical and horizontal dotted lines drawn from the position of the transmitter $(T x)$ divide the space into four quadrants. The solid edges of the rectangular objects have taken part in ray-object intersection test and the dotted edges have been discarded. It is seen from Fig. 1 (b) that there are eight objects in the area. However, only three of them are selected for each quadrant with respect to the ray emanated from the transmitter and four edges out of 12 edges of three objects are taking part in ray-object intersection test.

This study divides the whole space into four quadrants where the size of each quadrant is 90 degree. To trace the signals originated from the transmitter, the proposed IRT algorithm based on BAD is applied for those four quadrants individually. As can be observed from Fig. 2 (a), there are eight 
(a)

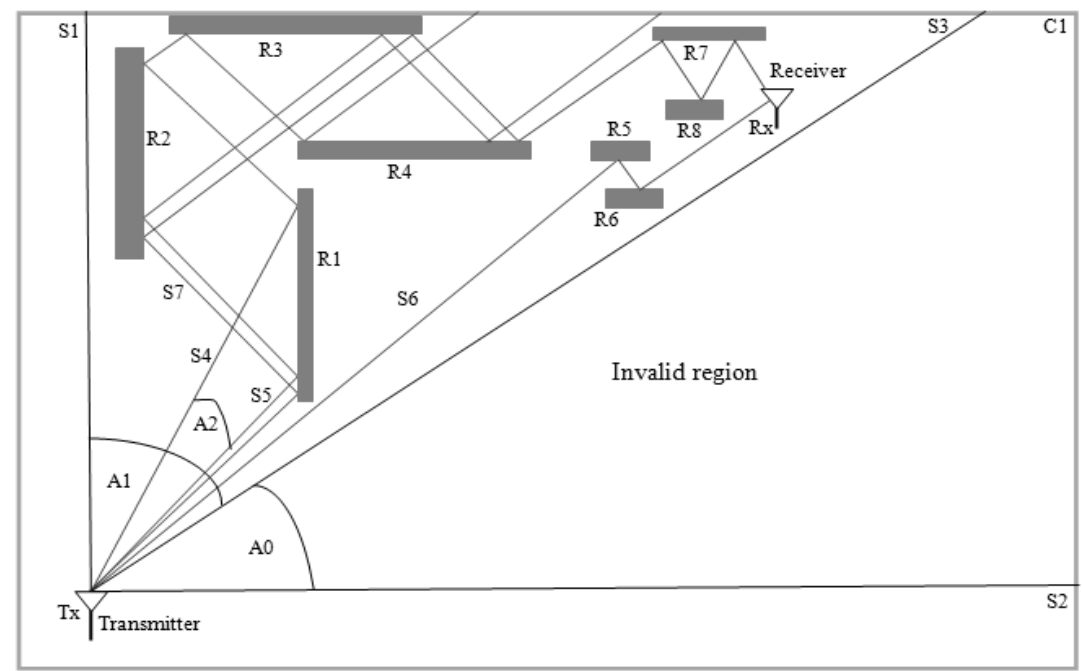

(b)

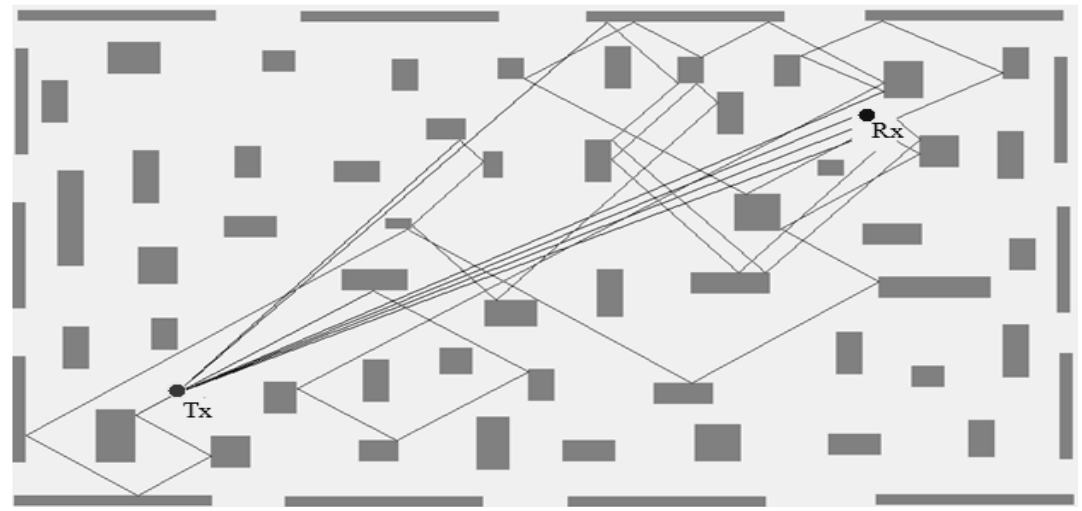

Fig. 2. (a) Radio signal prediction (b) Illustration of IRT in an indoor.

obstacles $R 1, R 2, \ldots, R 8$ (as reflectors). As in Fig. $2(\mathrm{a}), R x$ is in the first quadrant with respect to $T x$. Therefore, the proposed algorithm is applied on the first quadrant only to clarify its working principle. By launching two signals $S 1$ and $S 2$ from $T x$, a polygon consisting of the points $S 1, T x, S 2$, and $C 1$ is formed. The next step is to check if there is any obstacle within this polygon and thus considers only one point of each object, e.g., top left corner; and determines if the point lies on the interior of the polygon. If there is any object on the interior of the polygon, the angular distance between $S 1$ and $S 2$ is divided equally by launching another signal $S 3$. Consequently, two new polygons are formed for the regions $S 1 T x S 3$ and $S 3 T x S 2$, marked by $A 1$ and $A 0$, respectively where there is no object and no receiver exist within $A 0$. Hence, $A 0$ is marked as invalid region because all other signals within this area will be lost. However, the algorithm proceeds for the region $A 1$ as there are objects within this area. The signal $S 4$ is transmitted from the middle of the angular distance of the region $A 1$ and the path of the signal $S 4$ is followed until it passes away the propagation area. Here, the signal $S 4$ reflects on four obstacles $R 1, R 2, R 3$, and $R 4$, respectively and finally, passes away the indoor area. Another signal $S 5$ passes through the middle of the angular distance of the signals $S 3$ and $S 4$ and after reflection with the obstacles $R 1, R 2, R 3$, and $R 4$, passes away the propagation area. Here, 
the region $A 2$ is covered by the paths of the signals $S 4$ and $S 5$ contains no object. Hence, $A 2$ is also marked as invalid region as all other signals within this region will be lost. If the algorithm continues in this way, two significant signals $S 6$ and $S 7$ received by the receiver are found for the scenario of Fig. 2(a). A sample simulation of IRT is shown in Fig. 2(b), where the circles labeled by $T x$ and $R x$ represent the transmitter and the receiver and the rectangles are working as obstacles.

The radio signal prediction algorithm for an individual quadrant is as follows [1]: (i) Initiate the rays from the $T x$ at angles Start and End and trace the paths until becoming weaker or passing away the indoor environment or received by the $R x$ after reflections with the objects (to trace the path of the initiated ray from the $T x$, follow the path tracing as well as path building procedure); (ii) If any ray is received by the $R x$, consider it as a significant signal; (iii) Check the trajectories of the two signals emanated at angles Start and End, if they create valid polygon (if there is same number of reflections and corresponding reflectors are same, then the region covered by the paths of these two signals is a valid polygon); (iv) If the polygon is valid and there is no object within the polygon, skip the processing of all other signals within the angles Start and End, otherwise, continue to the next step; (v) Set Start $=$ Start and End $=($ Start + End $) / 2$ and go to the step (i); and (vi) Set Start $=($ Start + End $) / 2$ and End $=$ End and go to the step (i).

\section{Results and discussion}

To perform a fair comparison with the existing methods, a typical simulation environment of 10 different scenarios in Fig. 3 (a) has been used. Each simulation has been carried out between one $T x$ and one corresponding $R x$ of same numbering. The numbers set as labels of $T x$ and $R x$ in Fig. 3 (a) represent the number of scenarios. In Fig. 3 (b), the simulated results of the existing RT algorithms ("Ray-Tracing 1" refers to [2] and "Ray-Tracing 2" refers to [1]) along with the proposed IRT in terms of computation time have been presented and the proposed algorithm confirms the significant reduction of computation time from other algorithms. The proposed IRT algorithm identifies those objects that are in the same quadrant with respect to the current component of the path of the signal and have the possibility of being intersected with the signal. And, from those selected objects, only few edges of the objects are selected for ray-object intersection test. A comparison shown in Fig. 3(b) is based on the computation time that considers 10 different scenarios as presented in Fig. 3(a). The time is recorded in milliseconds (ms) and it is observed that the proposed IRT algorithm gives higher computational efficiency of about $25.65 \%$ and $51.5 \%$ in average than the existing RT techniques [1] and [2], respectively. Furthermore, the proposed IRT algorithm has been compared with the ray-launching algorithm $[3,4]$ in terms of signal prediction as shown in Fig. 3 (c). Here, two degree arbitrary angular increment has been considered for the ray-launching algorithm and it is found that the proposed algorithm achieves $115 \%$ better accuracy in average 
(a)

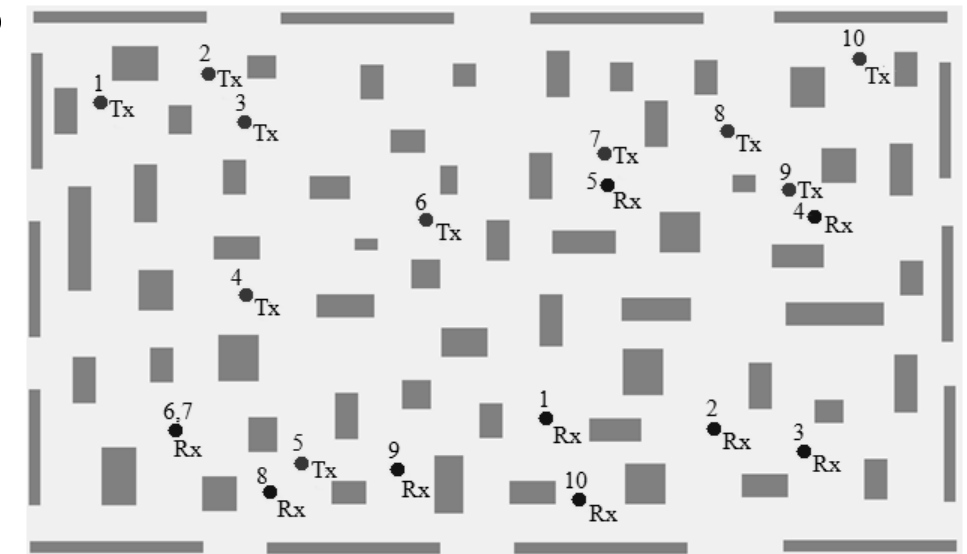

(b)

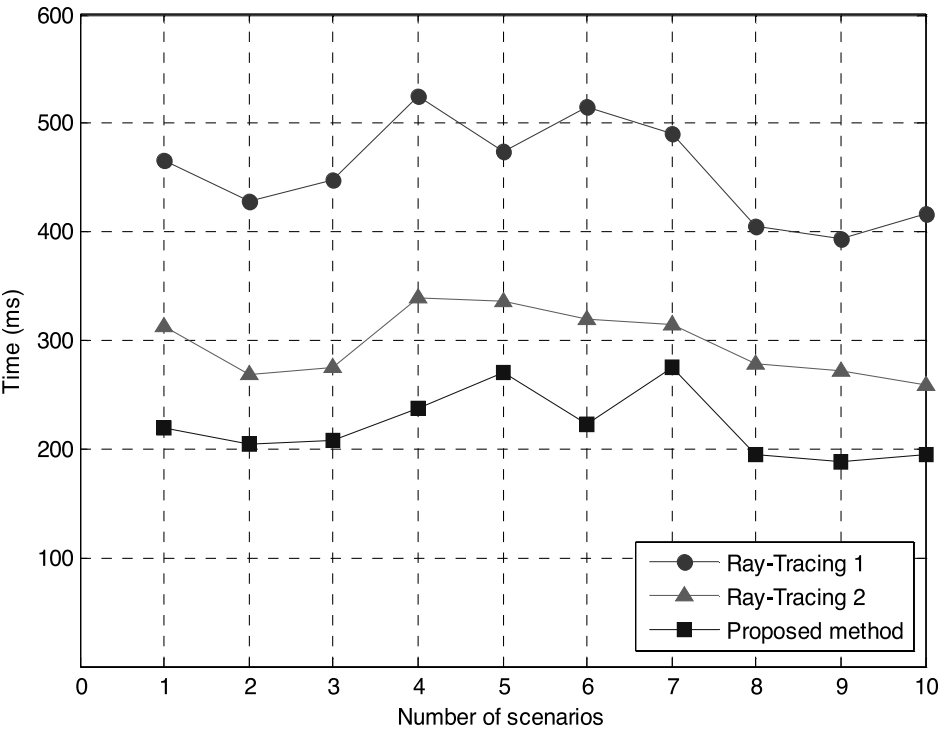

(c)

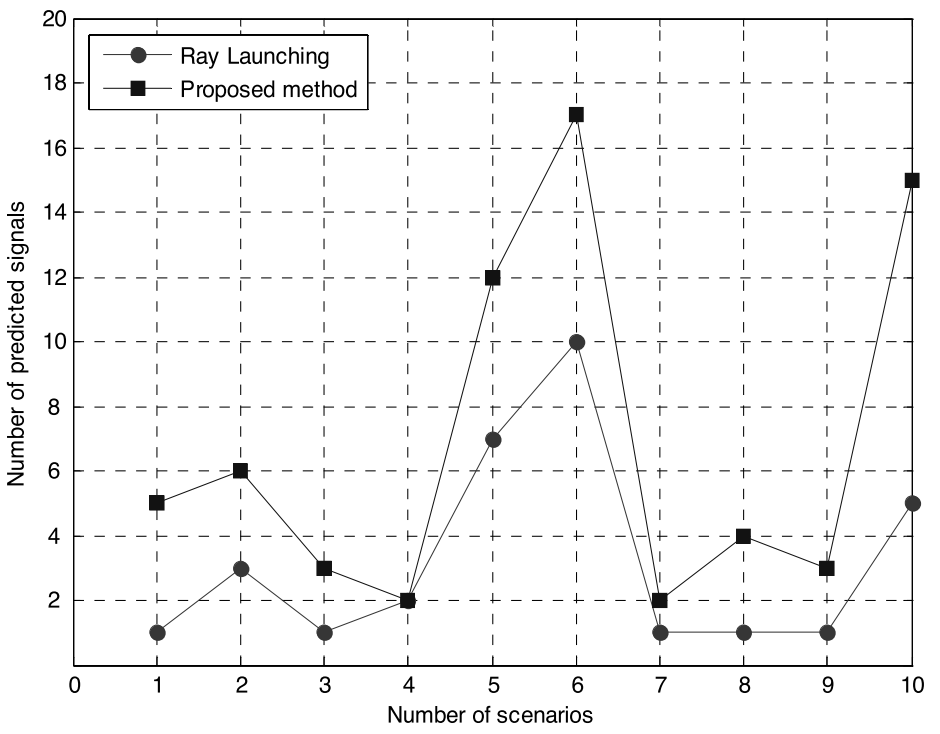

Fig. 3. (a) A simulation environment for performance evaluation (b) Comparison with existing RT techniques (c) Comparison with conventional raylaunching. 
in terms of signal prediction than the ray-launching algorithm for 10 different scenarios in Fig. 3 (a).

\section{Conclusion}

In this study, a novel IRT technique has been proposed that outperforms the existing RT and ray-launching algorithms. The obtained results show that the proposed IRT takes lower average time of $221.4 \mathrm{~ms}$ for 10 different scenarios while the existing RT techniques take $297.8 \mathrm{~ms}$ and $456.5 \mathrm{~ms}$, respectively. With respect to accuracy, the proposed method can predict higher number of signals (6.9 in average), while it is only 3.2 for the conventional ray-launching technique. 